\title{
Hyperdilution of CaHA fillers for the improvement of age and hereditary volume deficits in East Asian patients
}

This article was published in the following Dove Press journal: Clinical, Cosmetic and Investigational Dermatology

\section{Yates YY Chao' \\ Jong Woo $\mathrm{Kim}^{2}$ \\ JongSeo $\mathrm{Kim}^{3}$ \\ Hyungseok $\mathrm{Ko}^{4}$ \\ Kate Goldie ${ }^{5}$ \\ 'Chao and Chiu Institute of Aesthetic Medicine, Taipei, Taiwan; ${ }^{2} \mathrm{IU}$ Clinic, Seoul, Republic of Korea; ${ }^{3}$ Kim-JongSeo Plastic Surgery Clinic, Seoul, Republic of Korea; ${ }^{4}$ Samsung Lohas Clinic, Jeonju, Republic of Korea; ${ }^{5}$ European Medical Aesthetics Ltd, London, UK}

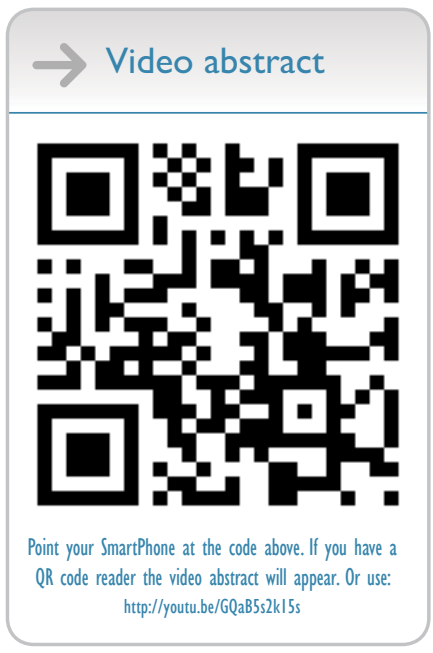

Correspondence: Yates YY Chao Chao and Chiu Institute of Dermatology 3/F 8 Chin Chen St, 105 Taipei, Taiwan Tel +886225454035

Email yateschao@gmail.com

\begin{abstract}
With their high elasticity and viscosity, calcium hydroxylapatite (CaHA) fillers are now widely used to treat age-related or hereditary facial soft tissue volume deficits. CaHA filler volume augmentation is further enhanced by its ability to stimulate neocollagenesis and improve skin quality. However, its high viscosity and cohesivity may hinder its spread and distribution, while its injection by cannula or needle may require moderate extrusion force and lead to uneven distribution or focal accumulation in tissues. Thus, new or modified delivery techniques and tools have emerged, particularly from East Asian physicians. One such technique is hyperdilution with diluents such as lidocaine or normal saline. CaHA hyperdilution appears to be more frequently used by experienced injectors who have varying methodologies. Here, we demonstrate the precise delivery of diluted filler to treat indications related to hereditary volume deficits, volume loss, or aging in the periorbital, nasolabial and submalar regions, marionette lines, and hollowed mid-facial areas. Regardless of age or indication, dilution eases filler delivery for the injector, while using lidocaine as the diluent decreases patient discomfort and minimizes pain. Increasing injection diluent volumes reduces filler thickness (viscosity) and facilitates its even spread, encouraging skin stimulation through more direct contact with tissues and minimizing unevenness. Our results effectively demonstrate that hyperdilution is an innovative and positive evolution in CaHA filler delivery.
\end{abstract}

Keywords: CaHA, hyperdilution, skin regeneration, facial sculpting, facial volumization

\section{Introduction}

The visible impact of aging is due partly to the loss of collagen and elastin fibers and the shifting distribution of fat due to reduced skin tone and aponeurotic tissue elasticity. Ultimately, these factors change the relationships between the various facial structures, causing wrinkles and skin folds. ${ }^{1,2}$ Using dermal fillers for soft tissue augmentation is a popular, minimally invasive aesthetic technique in East Asia. ${ }^{3-5}$ Calcium hydroxylapatite (CaHA) fillers, such as Radiesse ${ }^{\circledR}$ (RAD), have been used safely and effectively in soft tissue rejuvenation of face and body indications. ${ }^{6}$ RAD comprises CaHA microspheres within an aqueous carboxymethylcellulose gel carrier. ${ }^{7}$ Its small particle size imparts a smooth appearance beneath skin without visible filler beading. Both diluted and undiluted CaHA volumize tissues immediately and stimulate neocollagenesis ${ }^{8}$ within 2-3 months of treatment. Tissues treated with saline-diluted CaHA show higher levels of collagen type I, collagen type III, and elastin, indicating greater skin strength, elasticity, and viscoelasticity. ${ }^{9}$ Increases in angiogenic markers also suggest improved blood and nutrient flow, while a thicker dermis indicates sustained 
remodeling of the extracellular matrix. Its high density, viscosity, and cohesivity make it ideal for deep supraperiosteal injections in the malar eminence and zygomatic arches. ${ }^{10}$ Physicians have leveraged this capacity to increase dermal thickness and elastin production alongside its lifting ability to drive innovations in RAD use, such as filler hyperdilution to relieve pain and support skin quality improvements. The combination of $0.3 \%$ lidocaine with RAD (integrated into the next-generation product, $\operatorname{RAD}[+])^{11}$ now offers less painful procedures without having to manually mix the components. RAD hyperdilution with different diluents, in dilution ratios determined by injectors rather than manufacturers, has been documented. A 55-year-old patient with horizontal necklines was treated using diluted RAD. ${ }^{12}$ Contrary to the manufacturer's guidelines, RAD was mixed with $2 \%$ lidocaine and injected subdermally, resulting in positive outcomes even at 16-weeks postprocedure. A 3-tiered approach was also developed for the superperiosteal injection of premixed lidocaine and CaHA into malar and submalar areas and the piriform aperture. ${ }^{13}$ Here, we describe the novel and innovative, physician-driven use of RAD hyperdilution to rejuvenate aging-related facial indications in Asian patients.

\section{Patient case studies}

All patients were healthy Asian females, with no allergic history or underlying disease, presenting with aging-related aesthetic complaints and photographed at baseline, after treatment and/or at follow-up. Written informed consent and permission to publish case details and photographs were obtained from all patients.

\section{Case I: treatment of the periorbital, nasolabial, and submalar regions}

A 36-year-old female presented with infraorbital hollows, tear-trough volume loss, nasolabial fold and alar region recess, folliculitis, erythema, and enlarged pores. Augmentation by subdermal injection of CaHA filler was planned for the suborbicularis oculi fat layer, medial cheek, and nasolabial cheek fat pad. A $1.5 \mathrm{~mL}$ volume of CaHA was mixed with 0.3 $\mathrm{mL}$ of $2 \%$ lidocaine in a 5:1 dilution of filler. The filler was injected subdermally with a $23 \mathrm{G}, 50 \mathrm{~mm}$ blunt cannula into the nasolabial fold and fat pad using a fanning, sandwichlike technique. At the initial visit (Figure 1A), the patient received $2.6 \mathrm{~mL}$ of diluted filler on the right side and $2.2 \mathrm{~mL}$ of diluted filler on the left side. At 8 weeks (Figure 1B), skin texture and color were improved. At 8 months (Figure 1C), $1.8 \mathrm{~mL}$ of filler was injected into the right side of the face, and $1.3 \mathrm{~mL}$ of filler was injected on the left side. Marked improvements in skin tone and smoothness following treatment with diluted CaHA were not the original intention of the treatments. However, over a 14-month period and 3 additional follow-up sessions, further skin improvement,

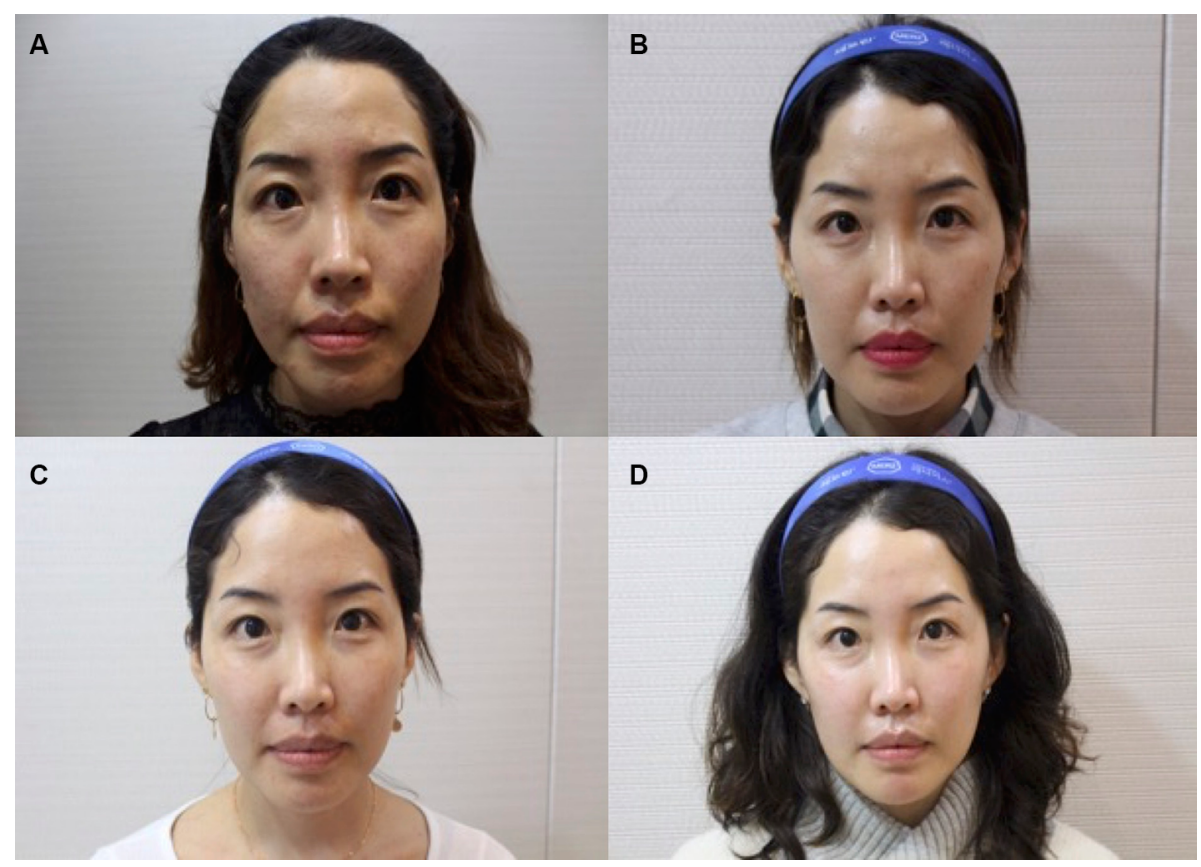

Figure I Treatment of periorbital, nasolabial, and submalar regions with $\mathrm{CaHA}$ filler results in volume augmentation and skin texture improvement.

Notes: Patient is shown at initial visit (A) prior to treatment and at follow-up visits at 8 weeks (B), 7 months (C), and I4 months (D) after the first procedure in which RAD CaHA filler was diluted with $2 \%$ lidocaine in a 5:I ratio. (Photos courtesy of Dr Jong Woo Kim.)

Abbreviations: CaHA, calcium hydroxylapatite; RAD, Radiesse ${ }^{\circledR}$. 
a rejuvenated appearance, and enhanced quality of the folliculitis, facial erythema, and enlarged pores also occurred without of the use of skin laser treatments or topical care products (Figure 1D).

\section{Case 2: treatment of marionette lines}

A 62-year-old female presented with pan-facial aging, including marionette lines ${ }^{14,15}$ with skin laxity, jowl fat accumulation, and skin drooping. A $0.5 \mathrm{~mL}$ volume of CaHA was diluted with $0.25 \mathrm{~mL}$ of $2 \%$ lidocaine, and $0.25 \mathrm{~mL}$ of normal saline. An entry point was created using a $23 \mathrm{G}$ needle at the lower part of the marionette line. The nondominant index finger guided filler injection into a superficial layer. $0.05 \mathrm{cc}$ of filler was injected at the approximate depth of the deep dermal to subdermal layer junction of the marionette lines with over 50 passes of a $27 \mathrm{G}, 40 \mathrm{~mm}$ cannula. The remaining filler $(0.05 \mathrm{cc})$ was injected with 50 passes into multiple points from the subdermal fat layer to the level above the marionette lines, the depressor labii inferioris and depressor anguli oris fat layers. Injecting a total of $1 \mathrm{cc}$ of diluted CaHA filler in over 100 passes, with up to $0.01 \mathrm{cc}$ of diluted CaHA filler per pass, was done to minimize lump and palpable mass formation. Immediately after injections, skin quality improvements were visible. At 1 month, biopsy of the injected area revealed fibroblasts surrounding CaHA particles (unpublished data) and the assessment by low-resolution derma-scope (Figure 3C and D) showed reduction of marionette lines, indicating that the filler corrected the volume loss (Figures 2B and C and 3B, D, and F). Fine wrinkles and skin fold improvements persisted for over 1 year (Figure 3 ).

\section{Case 3: mid-face treatment of volume loss and skin rejuvenation}

A 36-year-old female presented with thin, dry skin, decreased pan-facial skin elasticity anteriorly, and mid-face volume loss with consequent hollowed cheek and relative prominent zygoma and depressive temples (Figure 4A and C). Three milliliters of CaHA was mixed with $2 \%$ lidocaine in a $1: 1$ ratio (total injection volume of $6 \mathrm{cc}$ ) and injected with a $27 \mathrm{G}$ cannula into the subdermal layer of both cheeks. To treat a wide cheek area, up to $0.01 \mathrm{~mL}$ of filler per stroke was injected in a pattern of multiple passes to cover the whole cheek area. Following injection, volume restoration was immediately visible. At 5-months postinjection (Figure 4B and D), the patient's skin showed markedly improved skin tone, elasticity, and brightness (with less pigmentation). Morphological improvements included volume restoration of the hollowed cheek and camouflaging of prominent zygoma

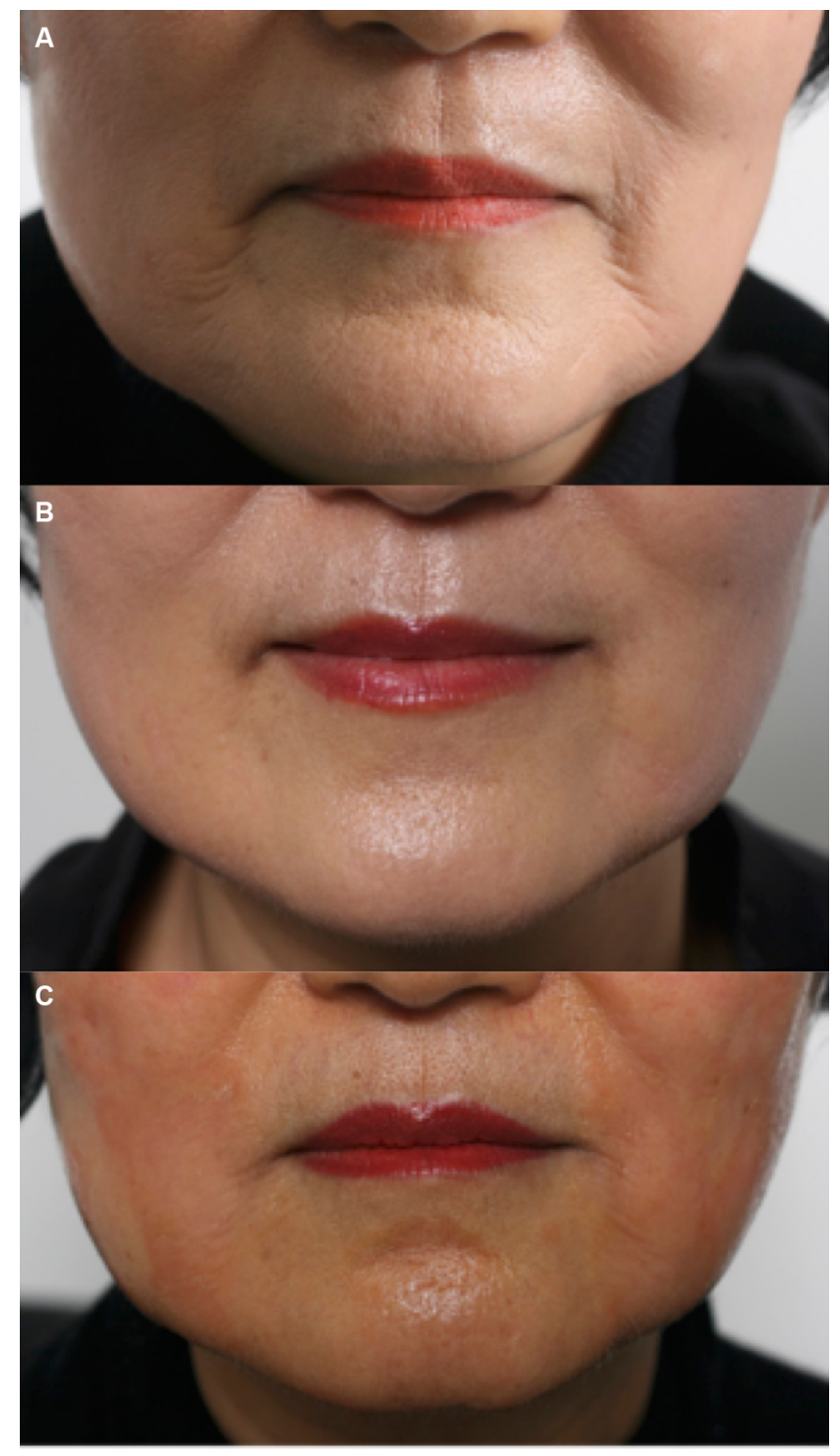

Figure 2 Treatment of marionette lines with diluted RAD using $27 \mathrm{G}$ cannula. Notes: Patient is shown at initial consultation (A) before treatment, I month after treatment (B), and I year (C) after treatment. Improvements to fine wrinkles were observed even after I month, due to the volumizing and neocollagenesis effect of RAD. (Photos courtesy of Dr Jongseo Kim.)

Abbreviation: RAD, Radiesse ${ }^{\circledR}$.

areas. Improvements in skin elasticity could be felt around the cheek area, resulting in a sharpened jawline and a more "lifted" look.

\section{Case 4: whole-face treatment by RAD hyperdilution}

A 58-year-old female presented with aging skin, signs of age-related collagen and elastin degeneration, forehead asymmetry with bony deficiencies, excess soft tissue on the left forehead (Figure 5), an abrupt zygoma-to-malar transition and distinct malar groove, jowls, and mid-face volume loss. To improve forehead skin quality, balance asymmetry, and 

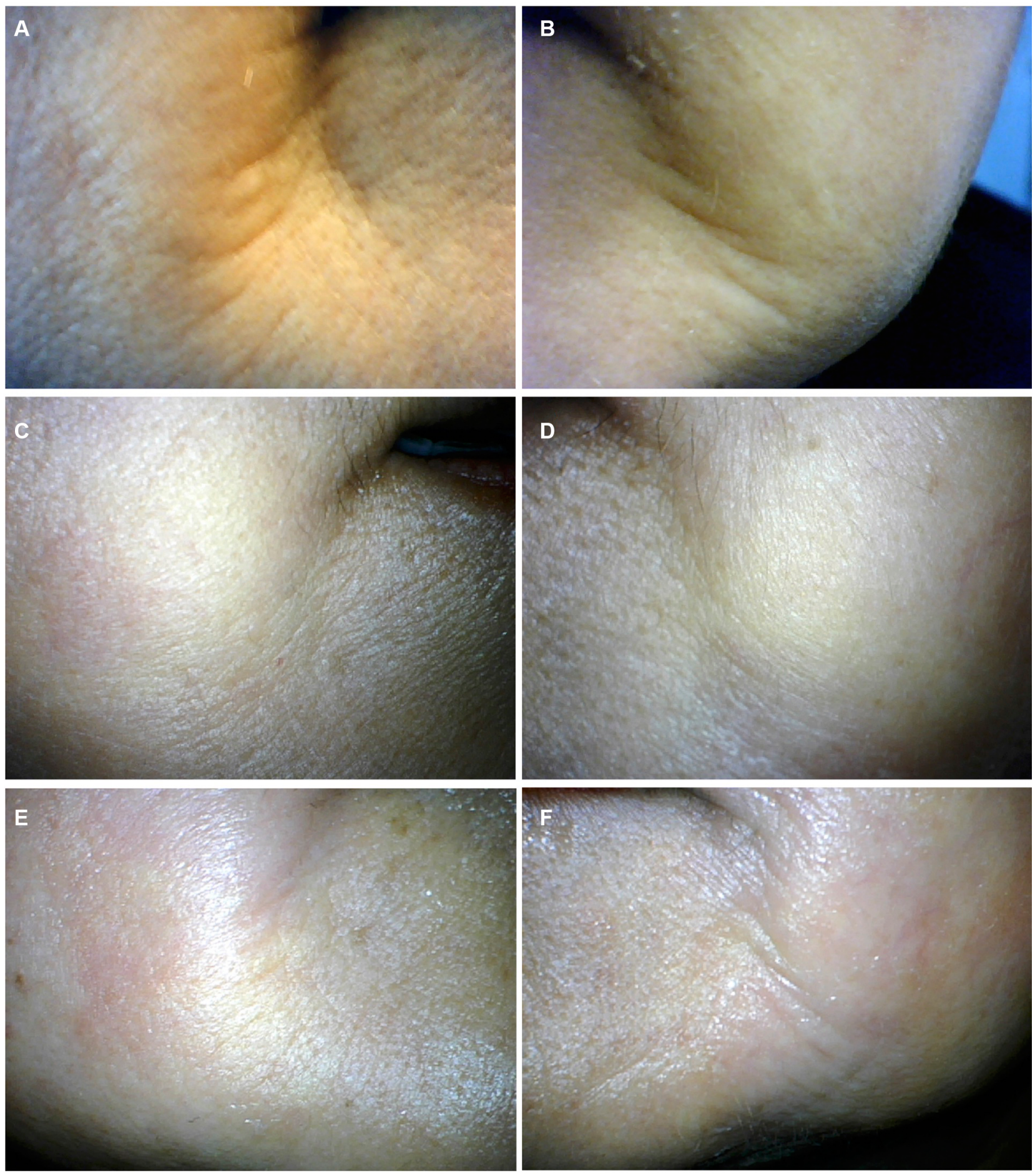

Figure 3 Low-resolution derma-scope images of marionette line area.

Notes: The left and right sides of the patient's face are shown at initial consultation before injection (A and B) and I month after treatment (C and $\mathbf{D})$. Improvements to deep and fine lines are visible and persist even after I year (E and F) due to neocollagenesis. (Photos courtesy of Dr Jongseo Kim.)

replace volume loss in the left frontal eminence and above the left superciliary arch, $1.5 \mathrm{~mL}$ of CaHA (diluted with $1.2 \mathrm{~mL}$ of normal saline and $0.3 \mathrm{~mL}$ of lignocaine) was injected subdermally using dermal scraping and a $25 \mathrm{G}$ cannula. Up to $0.01 \mathrm{~mL}$ of hyperdiluted filler was injected per pass with multiple passes. To augment temple volume loss and reshape brow prominence, $1.5 \mathrm{~mL}$ of diluted CaHA was injected into the inferomedial temple, between the superficial and deep temporal fascia. Up to $0.1 \mathrm{~mL}$ of filler was injected per pass in the brow. $0.01 \mathrm{~mL}$ of undiluted $\mathrm{RAD}(+)$ was injected per 


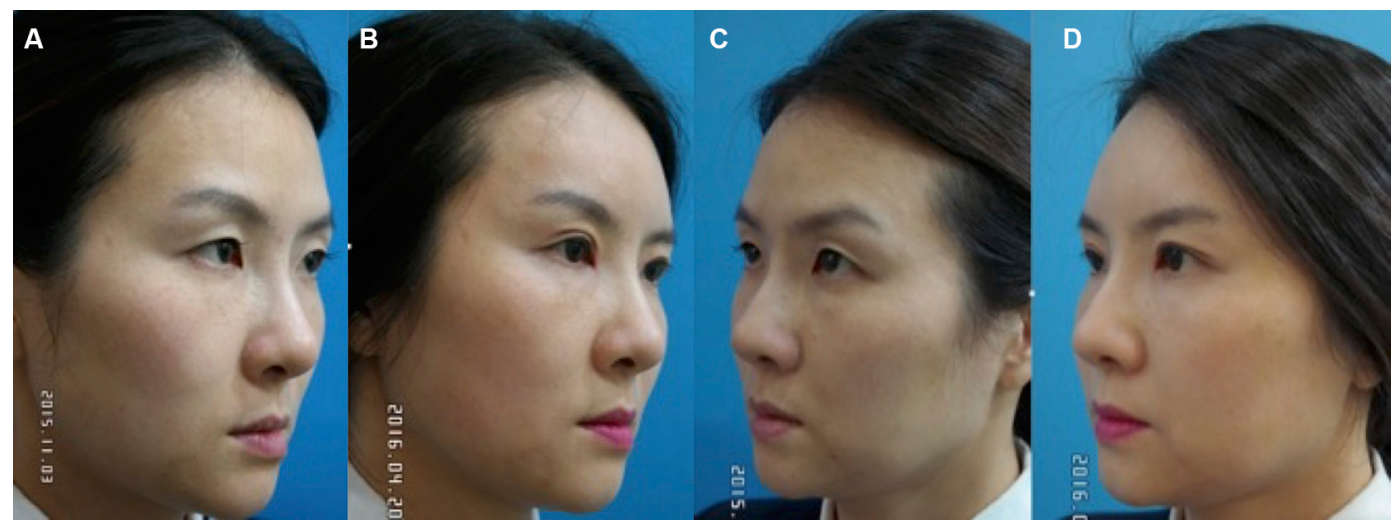

Figure 4 Treatment of mid-face volume loss with hyperdiluted RAD.

Notes: Patient is shown before treatment at initial visit (A and C), at 3.5 months (B), and 4.5 months (D) following RAD injection. (Photos courtesy of Dr Hyungseok Ko.) Abbreviation: RAD, Radiesse ${ }^{\circledast}$.

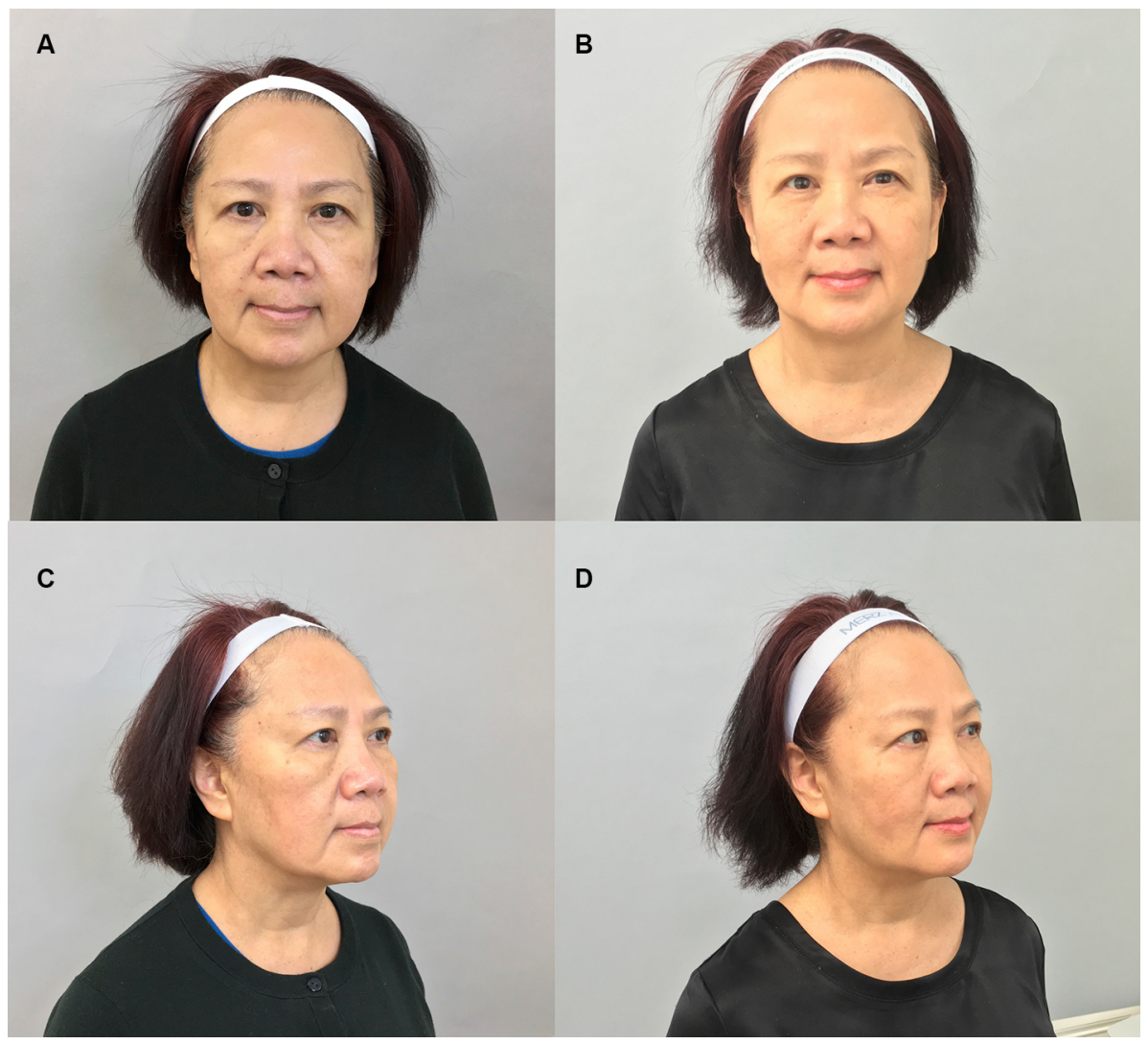

Figure 5 Treatment of the whole-face by RAD hyperdilution.

Notes: Patient is shown before (A and $\mathbf{C}$ ) and 3 months after treatment (B and $\mathbf{D})$. (Photos courtesy of Dr Kate Goldie.)

Abbreviation: RAD, Radiesse ${ }^{\circledR}$.

pass in retrograde fashion into superficial fat and subdermal layers into the mid-face for skin benefits and rebalancing of malar and submalar projection. Undiluted CaHA of $0.1 \mathrm{~mL}$ volume was injected per pass into submandibular areas in subdermal layers to build a straighter jawline, and $0.2 \mathrm{~mL}$ of undiluted RAD was injected subdermally for blending between the anterior and posterior areas. At 3 months, the forehead was rounder with better skin quality, and smoother forehead-to-temple, orbital ridge-to-zygoma, and zygomato-malar transitions were observed. The 2-layer approach and undiluted filler lifted the malar areas and smoothened the malar groove. Blending and sculpting of filler across the 
face also smoothened soft tissue transitions around the masseteric cutaneous ligament and volumized the lateral face.

\section{Discussion}

For many years, RAD has been used in Asia for facial volumization according to standardized manufacturer's treatment guidelines. The incorporation of lidocainehyperdiluted $\mathrm{CaHA}$ in our patient cases demonstrates a novel and innovative, physician-driven evolution of established delivery techniques. Importantly, the use of hyperdilution does not diminish the eventual level of aesthetic improvement from that seen with the neat product, although a large decrease in volume in the days immediately after treatment may be seen. The rationale and treatment goal associated with the use of diluted or undiluted fillers is also different; hence, a direct comparison of outcomes would not be appropriate. Nevertheless, maintaining an identical absolute amount of filler in the diluted treatment protocol to that in a neat protocol will ensure the same result, especially in terms of skin remodeling. As shown, the treatment of various aging-related indications resulted in immediate and satisfactory patient outcomes.

Aside from immediately visible structural improvements, CaHA fillers also enable long-term physiological improvements. Over time, gel resorption leaves only CaHA particles within the treated area, which facilitates histiocytic and fibroblastic activity, ${ }^{16}$ neocollagenesis, and elastin production in tissues surrounding the microspheres. ${ }^{9} \mathrm{CaHA}$ increases dermal thickness and angiogenesis, tightens the skin, and improves its texture. These benefits can and have been leveraged by physicians to fulfill different aesthetic goals in the same area of the face, with the use of just a single product. In older patients seeking treatment for skin laxity, drooping, and volume loss, the ability to offer a single minimally invasive intervention that produces numerous visible aesthetic improvements is desirable. When hyperdiluted, CaHA is easily applied as a dermal wash to further enhance skin regeneration without producing noticeable or unnecessary shape changes.

While dilution of filler facilitates its wider and more even distribution, caution is advised in areas such as the marionette lines, where gel injections may be difficult, or to avoid nodule formation and erythemas. Of note, the injection of diluted CaHA in our study was found to minimize the risks of irregularities. In marionette lines and more severe lines or conditions, a follow-up session may be required 3 weeks after the initial session, using the same volume of filler.
Dilution of CaHA with normal saline or other diluents makes the handling of superficial injections easier and facilitates filler maneuverability in deep tissue planes, while dilution with lidocaine reduces pain. Dilution also increases filler volume while decreasing viscosity, increasing spreadability, and lowering injection force from a needle or cannula. RAD and diluent should be mixed using at least 10 strokes inside a syringe ${ }^{17}$ with sufficient space to house the entire volume of diluted material, which ensures a more uniform syringe extrusion force.

Dilution creates a filler with increased moldability, versatility, maneuverability, and elasticity that also produces a more natural appearance immediately following injection. These modifications allow for more precise dosing and/ or superficial placement in the subdermal plane as a wash to stimulate neocollagenesis. Simultaneously, the volume of filler is redistributed from a localized to a wider area, permitting more even and finer filler distribution and structural lift for pan-facial treatments. CaHA dilution is also compatible with other treatment modalities and can be used in combination strategies. The ability to use a single product in different ways and for the dual goals of dynamic lifting and skin regeneration provides optimal and cost-effective patient offerings.

\section{Conclusion}

We effectively demonstrate that experimental innovations in the use of diluted CaHA in RAD include sculpting, contouring, and improving skin quality and texture to produce bespoke patient outcomes. Based on these limited case studies, lidocaine hyperdilution of RAD is safe and comfortable, and its added benefit of skin rejuvenation through neocollagenesis, increased elastin production, and angiogenesis affords sustained aesthetic rewards to patients originally seeking treatment solely for volume loss. As physicians acquire the knowledge and techniques to use this versatile product, we expect to see further developments in filler delivery, in particular in the innovation of tools such as injectors, or the combination use of needle with cannula.

\section{Acknowledgments}

The article processing charge was sponsored by Merz Asia Pacific. The authors express their appreciation to Merz Asia Pacific Pte Ltd for funding the preparation of this manuscript and to Shawna Tan for manuscript writing and editorial assistance. 


\section{Author contributions}

All authors contributed toward data analysis, drafting and revising the paper and agree to be accountable for all aspects of the work.

\section{Disclosure}

All authors have received an honorarium from Merz to attend a meeting. The authors report no other conflicts of interest in this work.

\section{References}

1. Baumann L. Skin ageing and its treatment. JPathol.2007;211(2):241-251.

2. Cogorno Wasylkowski V. Body vectoring technique with Radiesse ${ }^{\circledR}$ for tightening of the abdomen, thighs, and brachial zone. Clin Cosmet Investig Dermatol. 2015;8:267-273.

3. Carruthers J, Cohen SR, Joseph JH, Narins RS, Rubin M. The science and art of dermal fillers for soft-tissue augmentation. J Drugs Dermatol. 2009;8(4):335-350.

4. Emer J, Sundaram H. Aesthetic applications of calcium hydroxylapatite volumizing filler: an evidence-based review and discussion of current concepts: (part 1 of 2). J Drugs Dermatol. 2013;12(12):1345-1354.

5. 2012 Plastic Surgery Procedural Statistics [webpage on the Internet] 2012. Available from: http://www.plasticsurgery.org/news/plastic-surgerystatistics/2012-plastic-surgery-statistics.html. Accessed May 07, 2018.

6. Marmur ES, Phelps R, Goldberg DJ. Clinical, histologic, and electron microscopic findings after injection of a calcium hydroxylapatite filler. $J$ Cosmet Laser Ther. 2004;6(4):223-226.

7. Graivier MH, Bass LS, Busso M, Jasin ME, Narins RS, Tzikas TL. Calcium hydroxylapatite (Radiesse) for correction of the mid- and lower face: consensus recommendations. Plast Reconstr Surg. 2007;120(Suppl 6):55S-66S.
8. Amselem M. Radiesse ${ }^{\circledR}$ : a novel rejuvenation treatment for the upper arms. Clin Cosmet Investig Dermatol. 2016;9:9-14.

9. Yutskovskaya YA, Kogan EA. Improved neocollagenesis and skin mechanical properties after injection of diluted calcium hydroxylapatite in the neck and décolletage: a pilot study. J Drugs Dermatol. 2017;16(1): 68-74.

10. Eviatar J, Lo C, Kirszrot J. Radiesse: advanced techniques and applications for a unique and versatile implant. Plast Reconstr Surg. 2015;136(Suppl 5):164S-170S.

11. U.S. Food \& Drug Administration. Premarket Approval (PMA) [webpage on the Internet]. Silver Spring, MD: U.S. Food \& Drug Administration. Available from: http://www.accessdata.fda.gov/scripts/ $\mathrm{cdrh} / \mathrm{cfdocs} / \mathrm{cfpma} / \mathrm{pma} . c f m ? i d=P 050052 \mathrm{~S} 019$. Accessed June 10, 2016.

12. Chao YY, Chiu HH, Howell DJ. A novel injection technique for horizontal necklines correction using calcium hydroxylapatite. Dermatol Surg. 2011;37(10):1542-1545.

13. Lorenc ZP. A three-tiered approach for the use of calcium hydroxylapatite premixed with lidocaine for facial application. Cosmet Dermatol. 2012;25(6):266-270.

14. Dallara JM, Baspeyras M, Bui P, Cartier H, Charavel MH, Dumas L. Calcium hydroxylapatite for jawline rejuvenation: consensus recommendations. J Cosmet Dermatol. 2014;13(1):3-14.

15. Berlin AL, Hussain M, Goldberg DJ. Calcium hydroxylapatite filler for facial rejuvenation: a histologic and immunohistochemical analysis. Dermatol Surg. 2008;34(Suppl 1):S64-S67.

16. Holzapfel AM, Mangat DS, Barron DS. Soft-tissue augmentation with calcium hydroxylapatite histological analysis. Arch Facial Plast Surg. 2008;10(5):335-338.

17. Busso M, Voigts R. An investigation of changes in physical properties of injectable calcium hydroxylapatite in a carrier gel when mixed with lidocaine and with lidocaine/epinephrine. Dermatol Surg. 2008;34(Suppl 1):S16-S23.
Clinical, Cosmetic and Investigational Dermatology

\section{Publish your work in this journal}

Clinical, Cosmetic and Investigational Dermatology is an international, peer-reviewed, open access, online journal that focuses on the latest clinical and experimental research in all aspects of skin disease and cosmetic interventions. This journal is included on PubMed. The manuscript management system is completely online

\section{Dovepress}

and includes a very quick and fair peer-review system, which is all easy to use. Visit http://www.dovepress.com/testimonials.php to read real quotes from published authors 